\section{Angiographic pattern of recurrent choroidal neovascularization in age-related macular degeneration}

\begin{abstract}
Purpose To evaluate the angiographic characteristics of recurrent choroidal neovascularization ( $\mathrm{R}-\mathrm{CNV}$ ) in age-related macular degeneration (AMD).
\end{abstract}

Methods A prospective investigation on 107 consecutive patients with exudative AMD and CNV not involving the fovea was conducted. Fluorescein angiography (FA) and indocyanine green angiography (ICGA) were planned before krypton laser treatment, and after 3 weeks, 2, 3, 4, 6, 9, 12, 18, and 24 months from photocoagulation. Laser treatment was FA-guided in eyes with classic $\mathrm{CNV}$, and ICGA-guided in eyes with occult CNV on FA. Results At baseline on FA, $23.3 \%$ had classic CNV, whereas, $76.6 \%$ showed occult CNV. On ICGA, CNV assumed a focal and a plaque pattern in 81.3 and $18.6 \%$ of cases, respectively. Overall, post-laser CNVs occurred in 56 eyes. FA identified well-defined and ill-defined RCNV in 25 and $75 \%$ of cases, respectively. ICGA identified three different R-CNV patterns: focal, annular, and plaque. Focal RCNV was defined as a single dot-like hyperfluorescence, which was detected in $\mathbf{6 9 . 6 \%}$ of cases, with subfoveal location in half of them. Annular R-CNV was identified by a hyperfluorescent lesion, partially or completely encircling treated area, which was visible in $19.6 \%$ of cases, all with subfoveal involvement. Plaque R-CNV was defined as a hyperfluorescent lesion larger than 1 disc diameter in size, and was seen in $10.7 \%$ of cases, all with subfoveal location. Conclusions ICGA is able to improve R-CNV visualization identifying three different $R$ CNV patterns. Focal R-CNV is the most
M Battaglia Parodi, S Da Pozzo and G Ravalico

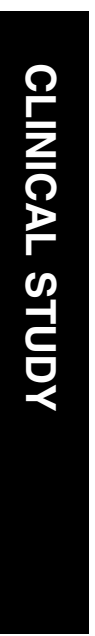

frequent pattern and can be re-treated in half of the cases.

Eye (2004) 18, 685-690. doi:10.1038/sj.eye.6701316

Published online 23 January 2004

Keywords: age-related macular degeneration, recurrent choroidal neovascularization, fluorescein angiography, indocyanine green angiography.

\section{Introduction}

Clinical trials demonstrated that laser photocoagulation is an effective treatment for choroidal neovascularization (CNV) in agerelated macular degeneration (AMD). ${ }^{1-4}$ Despite treatment, the major cause of photocoagulation failure is the development of recurrent choroidal neovascularization ( $\mathrm{R}-\mathrm{CNV}$ ), which has been reported in more than $50 \%$ of treated eyes. $^{5-7}$ Development of R-CNV in AMD is associated with higher incidence of severe visual loss compared with eyes that remain free from recurrences. ${ }^{6,7}$ Moreover, on fluorescein angiography (FA), R-CNV is ill-defined in 66$72 \%$ of cases ${ }^{8,9}$ and indocyanine green angiography (ICGA) is required to define recurrence location and extension. ${ }^{8-11}$

The most frequently reported pattern of R$\mathrm{CNV}$ on ICGA is a focal area of staining, commonly defined as a hot spot. ${ }^{10,11}$ Similar features have been described also for benign, temporary hot spots that can appear on ICGA during the first weeks after photocoagulation and resolve spontaneously without further treatment. ${ }^{9,12,13}$ These similarities can render the differentiation between temporary hot spots and either persistent or early R-CNV particularly awkward. Even though ICGA is
Eye Clinic, University of Trieste, Trieste, Italy

Correspondence: M Battaglia Parodi Eye Clinic University of Trieste Piazza dell'Ospedale 1 34129 Trieste, Italy Tel/fax: + 39040772449 E-mail:maubp@ yahoo.it

Received: 3 May 2003 Accepted in revised form: 18 August 2003 Published online: 23 January 2004 
commonly used in several centres to define presence, extension and site of R-CNV, a definite classification of RCNV pattern on ICGA is still not available.

The aim of the study is to describe the fluorescein and indocyanine green angiographic characteristics of $\mathrm{R}$ $\mathrm{CNV}$ in AMD.

\section{Methods}

A consecutive series of patients affected by exudative AMD and referred to the angiographic centre of the Eye Clinic of Trieste between December 1999 and March 2001 were prospectively considered.

Inclusion criteria were: age older than 50 years and diagnosis of exudative AMD with juxtafoveal or extrafoveal CNV.

Exclusion criteria were: presence of media opacities, previous laser treatment, or any other cause of $\mathrm{CNV}$, such as myopia, angioid streaks, presumed ocular histoplasmosis, chorioretinal inflammatory diseases, trauma, hereditary retinal disorders, and idiopathic CNV. Eyes presenting with polypoidal $\mathrm{CNV}$ and retinal vascular anomalous complex were also excluded. $\mathrm{CNV}$ showing ill-defined borders both FA on ICGA were also excluded from the study.

Diagnosis of exudative AMD was based upon the presence of visual acuity less than 20/20 with distortion on Amsler grid testing; drusen in both eyes; presence of retinal and/or subretinal haemorrhages, sensory retinal detachment, pigment epithelium detachment, and/or lipid exudation; detection of CNV on FA and/or on ICGA. Primary CNV was defined as newly diagnosed $\mathrm{CNV}$ that had never undergone any laser treatment before.

According to Macular Photocoagulation Study (MPS) reports, ${ }^{1-4}$ classic CNV was characterized by welldemarcated areas of early hyperfluorescence with progressive leakage in the late phases of FA, whereas occult CNV was characterized by ill-demarcated areas of hyperfluorescence on FA, with two major patterns: late leakage of undetermined source and fibrovascular pigment epithelium detachment.

$\mathrm{CNV}$ was judged to be either extrafoveal or juxtafoveal when the typical hyperfluorescence on FA was $200 \mu \mathrm{m}$ or more from the foveal centre, or 1-199 $\mu \mathrm{m}$ from the foveal centre, respectively.

On ICGA, focal CNV was defined as a well-delineated area of hyperfluorescence, less than 1 disc diameter in size, whereas plaque CNV corresponded to an area of hyperfluorescence larger than 1 disc diameter in size. Plaque CNV might show either well-defined or illdefined margins. ${ }^{14}$

As regards the postlaser angiographic examination, fluorescein angiograms showing an area of hyperfluorescence along the periphery of the treatment scar in the early transit phase with dye leakage during the later phases, were considered indicative of presence of CNV. Eyes in which FA showed such a fluorescein leakage within 6 weeks after laser treatment were classified as having persistent CNV. Eyes in which FA disclosed such a fluorescein leakage after the 6-week post-treatment visit were classified as having R-CNV.

On ICGA, the detection of a hyperfluorescent lesion lying on the margin of the treated area, showing a dye staining in the late phases of the examination was considered indicative of the presence of CNV. We considered three patterns of postlaser CNV on ICGA: focal, annular, and plaque.

Focal R-CNV was defined as a single dot-like hyperfluorescence along photocoagulated area border, detectable since early phases, but better visible during intermediate and late phases (Figure 1).

Annular R-CNV was defined as a hyperfluorescent irregularly shaped lesion, partially or completely encircling treated area, detectable during intermediate and late phases (Figure 2).

Plaque R-CNV was defined as a hyperfluorescent lesion larger than 1 disc diameter in size, expanding sectorially from the border of the treated area, and visible during intermediate and late phases (Figure 3).

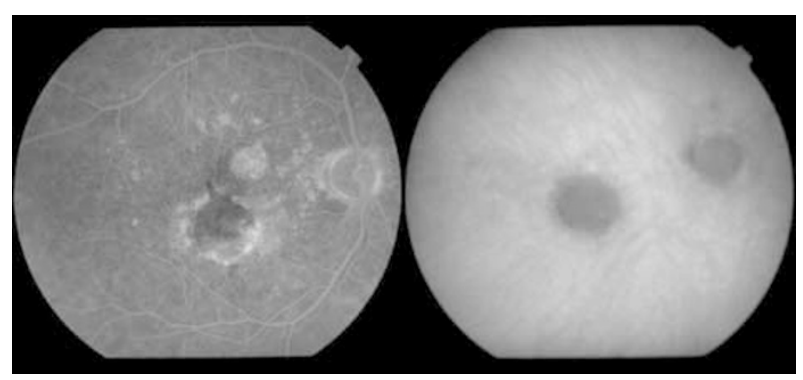

Figure 1 Focal R-CNV. Left: FA of occult R-CNV with subfoveal location. Right: ICGA of the same case revealing the focal R-CNV.

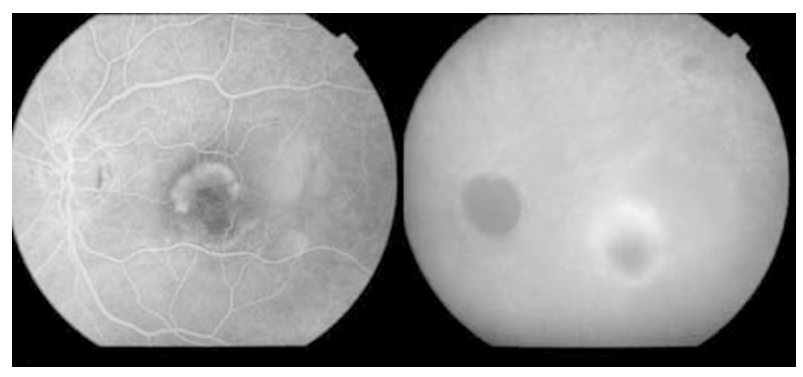

Figure 2 Annular R-CNV. Left: FA of R-CNV that appears as classic in the superior half and occult in the inferior half. Right: ICGA of the same case showing hyperfluorescence encircling the laser scar. 


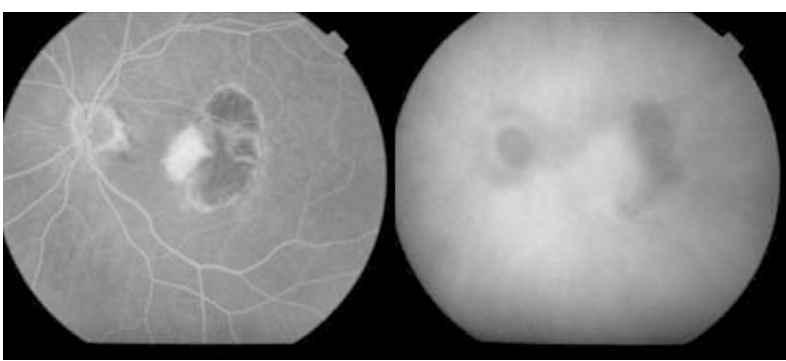

Figure 3 Plaque R-CNV. Left: Fluorescein angiography of RCNV that shows a mixed (classic and occult) pattern. Right: ICGA of the same case showing the whole extension of the R$\mathrm{CNV}$, which has a plaque pattern.

Eligible patients underwent an ophthalmological assessment including best-corrected visual acuity (BCVA) evaluation on standard ETDRS charts. BCVA values were expressed in $\log$ MAR. Examiners were unaware about patients' condition while measuring visual acuity.

FA and ICGA were performed on the IMAGEnet System H1024 (Topcon Corp.) the day before laser treatment, and then after 3 weeks after the laser photocoagulation. The cases disclosing a leaking marginal lesion on FA, or a hyperfluorescent lesion on the border of the treated area that showed staining in the late phases on ICGA, were re-evaluated in the subsequent week. When the 4-week examination showed the persistence or the enlargement of the leaking lesion on FA or of the hyperfluorescent lesion on ICGA, this feature was interpreted as indicative of the presence of persistent CNV. Further controls were planned 2, 3, 4, 6, $9,12,18$, and 24 months after photocoagulation.

Laser treatment was administered when the hyperfluorescent lesion detectable on FA and on ICGA diagnosed as $\mathrm{CNV}$ was associated with exudative phenomena detectable on biomicroscopy (ie blood, sensory retinal detachment, pigment epithelium detachment, lipid exudation) and the patients complained of metamorphopsia.

Laser photocoagulation was FA-guided for classic $\mathrm{CNV}$, and ICGA-guided for occult CNV assuming that the identified area of abnormal hyperfluorescence represents the full extent of the CNV. As mentioned above, we elected to exclude from the study all the CNV cases with ill-defined borders both on FA and on ICGA, even though they were not subfoveal and associated with exudative changes and subjective symptoms.

For ICGA-guided treatments, the hyperfluorescent area detected on ICGA was mapped onto the red-free frame using the computerized mapping program of the IMAGEnet System to allow treatment. Laser photocoagulation of primary $\mathrm{CNV}$ was carried out by a single author (MBP) by means of krypton red laser using a $200 \mathrm{~ms}$ duration, $200-\mu \mathrm{m}$ spot size and enough power to obtain a whitening of the retina, according to MPS guidelines.

After laser treatment, a post-treatment red-free frame was placed over the pre-laser angiogram to ensure the complete photocoagulation of the whole CNV.

Two authors (GR, SDP) independently evaluated angiographic features in order to diagnose the presence of persistent or R-CNV. A third author (MBP) was asked to act as arbitrator if an agreement could not be reached.

Each patient was fully informed about the purpose of research and provided signed informed consent to all procedures.

\section{Results}

A total of 112 out of 246 eyes (45. 5\%) fulfilled the inclusion and exclusion criteria. The remaining 134 were not considered to be amenable to laser treatment either because in 119 cases (48.3\%) the CNV was subfoveal, or because in 15 cases $(6 \%)$ the CNV showed ill-defined borders on both FA and ICGA.

Moreover, five patients were lost to follow-up (one patient died before the first control, and four patients refused further examination after laser treatment), and thus only 107 eyes of 107 patients were included in the study. Their mean age was $69.3+7.5$ years, with 64 female and 43 male patients.

At baseline on FA, 25 of 107 eyes (23.3\%) had classic CNV without any occult portion, whereas 82 eyes $(76,6 \%)$ showed occult CNV. In particular, fibrovascular pigment epithelium detachment was detected in 21 of 107 eyes $(19.6 \%)$ eyes, and late leakage of undetermined source in 61 eyes (57\%). In this latter group, 23 of $61(37.7 \%)$

showed also some evidence of classic component.

On ICGA, CNV assumed a focal pattern in 87 of 107 eyes $(81.3 \%$ ) (all 25 eyes with classic and additional 62 eyes with occult $\mathrm{CNV}$ on FA), and a plaque pattern in 20 eyes $(18.6 \%)$, all with well-defined borders. All 21 eyes presenting with occult $\mathrm{CNV}$ and fibrovascular pigment epithelium detachment were converted into focal CNV by ICGA.

The overlapping of postlaser hypofluorescent area on prelaser hyperfluorescent lesion showed that CNV was entirely covered by laser photocoagulation in all eyes.

Postlaser CNVs overall occurred in 53 of 107 eyes (49.5\%) at 12-month visit, and in 56 eyes (52.3\%) at 24 months after treatment, respectively. Figure 4 provides a Kaplan-Meier curve reporting the cumulative proportion of persistent and R-CNV within the follow-up.

In particular, five of 107 eyes (4.6\%) showed a leaking lesion on FA corresponding to a single hyperfluorescent lesion (hot spot) on ICGA at the 3-week control, which did not resolve at the 4-week control and was associated 


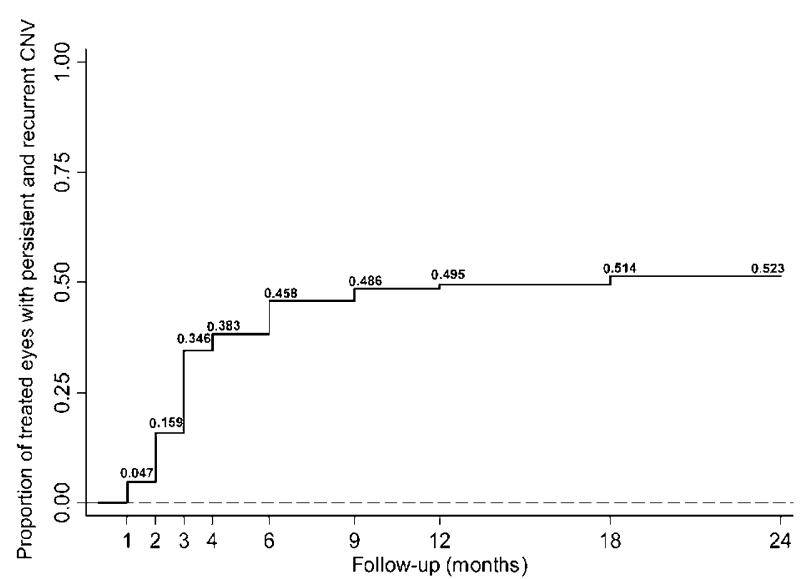

Figure 4 Kaplan-Meier curve reporting the cumulative proportion of persistent and recurrent CNV along the 24-month follow-up.

with persistence of exudative phenomena on biomicroscopy. These features were considered to be indicative of presence of $\mathrm{CNV}$, and thus, these five eyes were classified as having persistent CNV. Three of the above-mentioned five eyes showed an occult pattern on FA with uneven late leakage, whereas two revealed a classic pattern. On ICGA, all the five eyes showed a single hyperfluorescent spot along the margin of the treated area. Three persistent CNVs were subfoveal, whereas the remaining two were juxtafoveal and thus, were re-treated.

FA identified well-defined R-CNV in 14 of 56 eyes (25\%) and ill-defined R-CNV in 42 eyes (75\%). In the latter group, 16 eyes presented with a fibrovascular pigment epithelium detachment.

On ICGA, R-CNV was classified as focal in 39 of 56 eyes $(69.6 \%)$, with subfoveal location in 19 of 39 eyes (48.7\%), juxtafoveal in 12, and extrafoveal in eight eyes. Focal R-CNV appeared to be classic in 14 of 56 eyes (25\%) (eight of them subfoveal) and occult in 25 eyes (44.6\%) (11 of them subfoveal) on FA.

Annular R-CNV was identified on ICGA in 11 of 56 eyes (19.6\%), all with subfoveal involvement, presenting on FA as occult R-CNV in six cases and as mixed R-CNV (both classic and occult) in five cases.

Plaque R-CNV was visible on ICGA in six of 56 eyes $(10.7 \%)$. In these eyes, all R-CNVs had subfoveal location and on FA they were occult in five cases and mixed in one case.

The interobserver agreement ratio for $\mathrm{R}-\mathrm{CNV}$ diagnosis was $96 \%$.

Analysing the relationship between primary $\mathrm{CNV}$ and R-CNV patterns on ICGA, it can be noticed that focal $\mathrm{CNV}$ never recurred as plaque $\mathrm{R}-\mathrm{CNV}$, whereas plaque $\mathrm{CNV}$ never recurred as annular R-CNV (Table 1).
Table 1 Relationship between primary CNV pattern and RCNV pattern on ICGA

\begin{tabular}{lcccc}
\hline Primary CNV & \multicolumn{3}{l}{ Recurrent CNV (eyes) } & \\
\cline { 2 - 4 } & Focal & Annular & Plaque & Total \\
\cline { 2 - 4 } & 34 & 11 & 0 & 45 \\
Focal & 5 & 0 & 6 & 11 \\
Plaque & 39 & 11 & 6 & 56 \\
Total & & & & \\
\hline
\end{tabular}

Table 2 Mean BCVA (logMAR) at the time of R-CNV onset and at the end of the 24-month follow-up

\begin{tabular}{lccc}
\hline & Eyes (no.) & Onset & 24 months \\
\hline Focal & 39 & 0.66 & 0.94 \\
Annular & 11 & 1.18 & 1.66 \\
Plaque & 6 & 0.82 & 1.60 \\
\hline
\end{tabular}

At the time of its onset, focal R-CNV had the highest BCVA mean value among the three patterns (Table 2), retaining it up to the end of the follow-up. On the contrary, annular R-CNV had the worst baseline and final BCVA.

The mean BCVA in eyes with focal R-CNV turned out to be similar after the classification in three subgroups according to their location. In particular, the mean BCVA was $0.56,0.62$, and 0.7 in subfoveal, juxtafoveal, and extrafoveal focal R-CNV, respectively. Additional laser treatment was administered to the 12 juxtafoveal and eight extrafoveal focal R-CNVs, obtaining CNV closure in seven cases and subfoveal R-CNV in 13 cases. All eyes in this latter group showed well-defined borders on FA and focal pattern on ICGA.

\section{Discussion}

R-CNVs after conventional laser photocoagulation of primary AMD-related CNV are well-defined on FA only in almost one-third of cases, being ill-defined in the remaining two-thirds. ${ }^{8,9} \mathrm{~A}$ few studies have shown that ICGA is useful to define location and extension of illdefined R-CNV on FA in AMD. ${ }^{8-11}$ Nevertheless, even though ICGA is frequently employed in several centres for the detection of both primary $\mathrm{CNV}$ and R-CNV, a definite classification of the possible R-CNV patterns on ICGA is still lacking.

In the MPS report, postlaser CNVs have been identified in $66 \%$ of eyes following krypton laser photocoagulation within 2 years from treatment, ${ }^{7}$ whereas in our study the postlaser $\mathrm{CNV}$ occurrence at 24 months is $52.3 \%$. Overall, our cumulative proportion of persistent and R-CNV within 24 months is lower with respect to the MPS. Actually, a comparison with MPS is 
not possible because several aspects differed, most of all because in our consecutive series of patients only 25 out of 107 eyes (23.3\%) had classic pattern on FA at baseline. Moreover, in our investigation, laser treatment was FAguided for classic CNV and ICGA-guided for new vessels with occult features on FA, assuming that the identified area of abnormal hyperfluorescence represents the whole extent of the CNV. Lastly, the identification of persistent $\mathrm{CNV}$ and R-CNV was based not only on FA, but also on ICGA findings, with overlapping of post-treatment red-free frame on the prelaser angiogram to ensure the complete photocoagulation of the whole CNV.

The R-CNV occurrence in other series of patients examined by means of ICGA appears to be highly variable, ranging from 28 to $97 \%,{ }^{8-12,15}$ with huge differences as for types of the lesion analysed, photocoagulation modality, definition of R-CNV, and, most of all, duration of the follow-up.

In accordance with previous reports, ${ }^{8,9}$ we detected occult R-CNV on FA in $76 \%$ of cases, but more interestingly, we identified three different R-CNV patterns on ICGA. Focal R-CNV is the most frequent pattern, accounting for two-thirds of cases, having its origin both in primary focal and plaque CNV. Originally reported as hot spot by Sorenson, who recommended its prompt photocoagulation, ${ }^{10,11}$ focal R-CNV is certainly the best known R-CNV pattern on ICGA.

In almost $20 \%$ of cases, R-CNV shows features of an annular type with foveal involvement in all cases, originating from focal primary CNV. Reichel et $\mathrm{al}^{8}$ described a similar pattern with circumferential hyperfluorescence detectable since $3-5 \mathrm{~min}$ after dye injection and interpreted it as a plaque CNV.

Nevertheless, in our experience, annular R-CNV turns out to be detectable during intermediate and late ICGA phases, including new vessels imaged both as occult and as mixed on FA.

Plaque R-CNV is the less frequent type, invariably with foveal involvement and originates only from primary plaque $\mathrm{CNV}$.

Annular and plaque R-CNVs typically involve the fovea, rendering an additional laser photocoagulation of the lesion not feasible without damaging the fovea. As focal R-CNVs are regarded, half of the cases turn out to be juxtafoveal or extrafoveal at the time of diagnosis and are thus re-treatable, even in centres not inclined to treat subfoveal R-CNV by conventional photocoagulation. In particular, ill-defined R-CNV on FA was converted on ICGA into a focal R-CNV in 23 cases. Of these 23 cases, 13 $(56 \%)$ were nonsubfoveal and thus, amenable to laser retreatment. This finding is in accordance with previous observations and confirms that ICGA is a useful adjunctive technique to increase the number of patients eligible for additional laser treatment. ${ }^{8-11}$
When visual function is taken into consideration, focal R-CNV has the best visual acuity at the time of its onset and annular R-CNV the worst. This behaviour is preserved up to the end of the 24-month follow-up. The relatively good visual acuity of focal R-CNV at its onset might be due to the coexistence of subfoveal and nonsubfoveal CNV within the focal R-CNV category, but a subdivision into the three groups indicates that at the time of its onset subfoveal focal R-CNV still has a better visual acuity than both subfoveal annular and subfoveal plaque R-CNV. We guess that this feature could be related to the smaller size of the focal R-CNV that probably allows a more stable fixation in a near retinal locus.

Focal R-CNV shows a better BCVA in comparison with annular and plaque R-CVN even at the end of the followup. This result probably depends also on the positive effect of the laser re-treatment, which has been carried out in the half of the cases.

Focal R-CNV may be difficult to differentiate from the spontaneously resolving hot spots visible during the first-weeks postlaser angiographic examinations. ${ }^{9,12,13}$ In our experience, only when the hot spot is detectable in the early ICGA phases along the course of a retinal or choroidal vessel, we can interpret it as the result of a thermal vasculitis. Otherwise, the angiographic examinations can be confusing. Indeed, ICGA examination carried out too early may create interpretative doubts, but an evaluation performed too late may lead to an unacceptably high ratio of subfoveal recurrent CNV. On the basis of our previous study, ${ }^{13}$ we routinely perform the first postlaser angiographic control 3 weeks after treatment, and we strictly control in the subsequent week the patients showing a marginally leaking lesion on FA, or a hot spot on ICGA, in order to define the nature of the lesion. The persistence of the leaking lesion on FA or the enlargement of the hot spot are indicative of the presence of $\mathrm{CNV}$, which should be re-treated if it is nonsubfoveal.

We are aware that our report has some shortcomings. First of all we took into consideration only primary CNVs that were not subfoveal and showed well-defined margins on ICGA. In this way, we ruled out all the subfoveal CNVs, especially the mixed forms showing on ICGA the coexistence of plaque CNV with hot spots.

These selection criteria could explain both the high rate of primary CNV with focal pattern on ICGA and the low rate of plaque $\mathrm{CNV}$ without subfoveal involvement taken into consideration for the study. Possibly other R-CNV patterns might be detectable from photocoagulation of these CNV subgroups.

Second, our analysis could be limited by the employment of a fundus camera-based system. Scanning laser angiography systems, thanks to their different 
characteristics, could unveil more accurately persistent or early recurrent $\mathrm{CNV}$ from early phases of examination, showing different patterns.

Moreover, the number of cases is too small, especially as annular and plaque $\mathrm{R}-\mathrm{CNV}$ are regarded, to allow an accurate subgroup analysis.

Last, laser photocoagulation was ICGA-guided for occult CNV on FA, based on the assumption that hyperfluorescent lesion can be regarded as CNV.

In conclusion, the present study shows that ICGA is able to improve R-CNV visualization, since R-CNVs are ill-defined in almost two-thirds of cases on FA. Three different R-CNV patterns can be identified by ICGA: focal, annular, and plaque. Focal R-CNV is the most frequent pattern, appearing in $69.6 \%$ of cases. Focal R$\mathrm{CNV}$ turns out to be nonsubfoveal in half of the cases at the time of diagnosis, allowing an additional laser treatment without damaging the fovea.

\section{Acknowledgements}

We thank Dr J Slakter and Dr G Virgili for their helpful comments.

\section{References}

1 Macular Photocoagulation Study Group. Krypton laser photocoagulation for neovascular lesions of age-related macular degeneration: results of a randomized clinical trial. Arch Ophthalmol 1990; 108: 816-824.

2 Macular Photocoagulation Study Group. Argon laser photocoagulation for neovascular maculopathy: five-year results of a randomized clinical trials. Arch Ophthalmol 1991; 109: 1109-1114.

3 Macular Photocoagulation Study Group. Laser photocoagulation for juxtafoveal choroidal neovascularization: five-year results from randomized clinical trials. Arch Ophthalmol 1994; 112: 500-509.

4 Macular Photocoagulation Study Group. Laser photocoagulation of subfoveal neovascular lesions of age-related macular degeneration: updated findings from two clinical trial. Arch Ophthalmol 1993; 111: 1200-1209.

5 Sorenson JA, Yannuzzi LA, Shakin JL. Recurrent subretinal neovascularization. Ophthalmology 1985; 92: 10591074.

6 Macular Photocoagulation Study Group. Recurrent choroidal neovascularization after argon laser photocoagulation for neovascular maculopathy. Arch Ophthalmol 1986; 104: 503-512.

7 Macular Photocoagulation Study Group. Persistent and recurrent choroidal neovascularization after krypton laser photocoagulation for neovascular lesions of age-related macular degeneration. Arch Ophthalmol 1990; 108: 825-831.

8 Reichel E, Pollock DA, Duker JS, Puliafito CA. Indocyanine green angiography for recurrent choroidal neovascularization in age-related macular degeneration. Ophthalmic Surg Lasers 1995; 26: 513-518.

9 Regillo CD, Blade KA, Custis PH, O'Connell SR. Evaluating persistent and recurrent choroidal neovascularization. The role of indocyanine green angiography. Ophthalmology 1998; 105: 1821-1826.

10 Sorenson JA, Yannuzzi LA, Slakter JS, Guyer DR, Ho AC, Orlock DA. A pilot study of indocyanine green videoangiography-guided laser treatment of recurrent occult choroidal neovascularization in age-related macular degeneration. Arch Ophthalmol 1994; 112: 473-479.

11 Sorenson JA, Yannuzzi LA. Recurrent occult choroidal neovascularization. In: Yannuzzi LA, Flower RW, Slakter JS (eds). Indocyanine Green Angiography. Mosby: St Louis, MO, 1997, pp 217-230.

12 Chen CJ, Chen LJ, Miller KR. Clinical significance of postlaser indocyanine green angiographic hot spots in age-related macular degeneration. Ophthalmology 1999; 106: 925-931.

13 Parodi MB, Da Pozzo S, Ravalico G. Early angiographic changes after laser treatment of choroidal neovascularisation in age-related macular degeneration. Graefes Arch Clin Exp Ophthalmol 2001; 239: 900-908.

14 Yannuzzi LA, Slakter JS, Sorenson JA, Guyer DR, Orlock DA. Digital indocyanine green videoangiography and choroidal neovascularization. Retina 1992; 12: 191-223.

15 Da Pozzo S, Parodi MB, Ravalico G. A pilot study of ICG-guided laser photocoagulation for occult choroidal neovascularization presenting as a focal spot in age-related macular degeneration. Int Ophthalmol 2001; 24: 187-194. 